\title{
2D magnetism gets hot
}

\author{
The demonstration of room-temperature ferromagnetism in an atomically thin layer broadens the prospects for \\ device applications of 2D van der Waals materials.
}

S oon after the first successful exfoliation of graphene in $2004^{1}$, scientists started dreaming of devices made from purely two-dimensional (2D) materials. These dreams are based on appealing properties, such as high flexibility, optical transparency or enormous carrier mobility, of layered van der Waals materials when sliced to the monolayer limit. The ability to produce micron-scale flakes with vanishing defect concentration and to combine different properties in single devices by simply stacking flakes of various materials on top of each other has fuelled these dreams further.

Since then, researchers have established functionalities such as single-photon emission or photon-to-charge conversion. Intrinsic magnetic order in a single 2D layer, however, was still missing from the $2 \mathrm{D}$ portfolio until recently, but has been wished for in view of lowpower ultra-compact spintronic applications.

In early 2017, two independent experiments showed magnetic order in two different systems at low temperature down to the very limit of $2 \mathrm{D}$. Gong and co-workers showed that in the Heisenberg-type ferromagnet $\mathrm{Cr}_{2} \mathrm{Ge}_{2} \mathrm{Te}_{6}$ the Curie temperature is suppressed when crossing from $3 \mathrm{D}$ to $2 \mathrm{D}$, but ferromagnetic order was detected down to the bilayer system ${ }^{2}$. Meanwhile, Huang et al. showed that in $\mathrm{CrI}_{3}$, even the monolayer shows Ising-type order and magnetization stable up to $45 \mathrm{~K}\left(\right.$ ref. $^{3}$ ). However, two monolayers of $\mathrm{CrI}_{3}$ combined into a bilayer lack a net magnetization because of an antiferromagnetic coupling between the two layers. These 2D magnets provide a playground to study new phenomena in low-dimensional magnetism, create new topological phases ${ }^{4}$ or break time-reversal symmetry in 2D stacks for valleytronics ${ }^{5}$. While low-temperature $2 \mathrm{D}$ magnets are precious for proof-of-principle devices, most technological applications will demand magnetic order at room temperature. Bonilla et al. have now tackled this challenge. In this issue, the authors demonstrate ferromagnetic order in monolayers of $\mathrm{VSe}_{2}$ grown on either graphite or $\mathrm{MoSe}_{2}$ (ref. ${ }^{6}$ ). Beside the fact that this transition metal dichalcogenide is paramagnetic in bulk, these results are remarkable because the ferromagnetic order with large magnetic moments persists above room temperature.
But where will we go from here? Recent experiments have demonstrated all-electric reversible control of the magnetization in bilayer $\mathrm{CrI}_{3}\left(\right.$ ref. $^{7}$ ). In addition, single-layer $\mathrm{CrI}_{3}$ emits circularly polarized photons when excited with linearly polarized light, and the helicity then depends on the magnetization direction of the layer ${ }^{8}$. It might not take too long until the first room-temperature $2 \mathrm{D}$ spin valves or flexible single-photon sources with electric polarization control are demonstrated. We are not there yet and hurdles for industrial-scale applications are substantially higher, but the toolbox for engineers has been updated.

Published online: 10 April 2018

https://doi.org/10.1038/s41565-018-0128-9

\section{References}

1. Novoselov, K. S. et al. Science 306, 666-669 (2004).

2. Gong, C. et al. Nature 546, 265-269 (2017).

3. Huang, B. et al. Nature 546, 270-273 (2017).

4. Tse, W.-K. et al. Phys. Rev. B 83, 155447 (2011).

5. Zhong, D. et al. Sci. Adv. 3, e1603113 (2017).

6. Bonilla, M. et al. Nat. Nanotech. https://doi.org/10.1038/s41565018-0063-9 (2018).

7. Jiang, S., Shan, J. \& Mak, K. F. Nat. Mater. https://doi.org/10.1038/ s41563-018-0040-6 (2018).

8. Seyler, K. L. et al. Nat. Phys. 14, 277-281 (2018).

\section{Quantum dots on your mind}

\section{Colloidal quantum dots can provide important information on electrical activity in neurons despite the difficulty of in vivo experiments.}

( ur memories, our thoughts and our actions can eventually be traced down to the combination of elementary electric and chemical signals. But monitoring the connections among 100 billion neuron cells is at the very least a daunting endeavour. The first step to solving the brain puzzle consists of the monitoring of electric signals emitted in micron or submicron regions and that vary in time on the scale of milliseconds. A number of nanostructures could be suited for this purpose and, especially encouraged by the launch of the BRAIN Initiative by the Obama administration, nanotechnology researchers have demonstrated various ways in which electrical signals can be recorded.

Perhaps the most successful approach so far has been that of using nanowires, isolated but also in arrays, as electrodes ${ }^{1}$, with the aim of obtaining direct measurements of neuron electrical activity. While a range of promising results has been achieved, a number of challenges are still present, the most notable being the invasiveness of the nanoprobes, a property that makes them likely to modify the tissue they are supposed to sense ${ }^{2}$.

In a Perspective published in this issue, Alexander Efros and co-authors examine potentially less invasive approaches based on colloidal quantum dots (QDs) ${ }^{3}$. These semiconductor nanoparticles are only a few nanometres across, which is comparable to the thickness of the neuron membrane. Both the intensity and wavelength of the fluorescence of a QD are affected by a time-dependent electric field. Experiments have shown that QD luminescence can trace a voltage spike similar to that associated with a firing neuron ${ }^{4}$. However, QDs should be placed directly inside the neuron membrane, which is challenging for a variety of fundamental reasons. A different strategy is to place the QD outside the membrane and link it to a small donor centre located inside. In this case, the electric field variation affects the donor centre and hence indirectly affects the luminescence of the linked QD. Importantly, this approach has been demonstrated in vivo ${ }^{5}$.

Though highlighting the first successes, the Perspective has the merit of avoiding hyping their implications. Rather, it provides estimates of the optical requirements necessary for realistic experiments and highlights the numerous challenges, leading to the conclusion that in vivo electrical sensing with QDs is a long-term goal. However, rather than taking this as a negative outcome, we should simply concentrate on the important insight that these nanoparticles can provide through in vitro experiment.

Published online: 10 April 2018 https://doi.org/10.1038/s41565-018-0129-8

\footnotetext{
References

1. Spira, M. E. \& Hai, A. Nat. Nanotech. 8, 83-94 (2013).

2. Zhang, A. \& Lieber, C. M. Chem. Rev. 116, 215-257 (2016).

3. Efros, A. I. et al. Nat. Nanotech. https://doi.org/10.1038/s41565018-0107-1 (2018).

4. Rowland, C. E. et al. Nano Lett. 15, 6848-6854 (2015).

5. Nag, O. K. et al. ACS Nano 11, 5598-5613 (2017).
} 